\title{
Factores pronósticos médicos y sociales asociados a urolitiasis en pacientes sometidos a nefroureterolitotomía láser
}

\section{Medical and social prognostic factors associated with urolithiasis in patients undergoing flexible ureteroscopy and laser lithotripsy}

\author{
Andres Quiñones ${ }^{1} \quad$ Juliana Arenas $^{1}$ Nicolás Fernández ${ }^{1}$ \\ 1 Unidad de Urología, Hospital Universitario San Ignacio, Pontificia \\ Universidad Javeriana, Bogotá, Colombia \\ Urol Colomb 2018;27:67-73.

\begin{abstract}
Address for correspondence Andres Quiñones, Unidad de Urología Hospital Universitario San Ignacio, Pontificia Universidad Javeriana, Bogotá, Colombia (e-mail: andresfelipeqr85@hotmail.com; quinones.andres@javeriana.edu.co).
\end{abstract}

\section{Resumen}

Palabras clave

- nefrolitotomía láser

- ureterolitotomía láser

- urolitiasis

- complicaciones

- factores de riesgo

- escalas de predicción de riesgo
Objetivos La fibróptica láser se ha convertido con el paso de los años en el procedimiento de elección en la mayoría de los casos de urolitiasis; sin embargo, se han descrito pocas escalas de predicción de riesgo y las que hay no incluyen el tiempo en el que se hace este procedimiento. Nuestro objetivo es hacer una descripción sobre los factores que influencian los desenlaces relacionados con las complicaciones de esta cirugía.

Materiales y métodos Se incluyó a 149 pacientes con patología litiásica a quienes se les realizó nefroureterolitotomía retrógrada con fragmentación láser entre los años 2012 y 2015. Se analizaron variables asociadas a las características del cálculo, la anatomía, el tiempo entre diagnóstico y la intervención y las complicaciones pre, intra y postoperatorias. Se diseñó una herramienta de recolección sobre la base de las historias clínicas y se analizó con medidas de tendencia central y de asociación.

Resultados Se encontró una correlación positiva entre las complicaciones preoperatorias, intraoperatorias y especialmente en las postoperatorias con el número de días orden-cirugía, con una pendiente con tendencia positiva a medida que se intervienen los pacientes más tardíamente. En las variables relacionadas con el índice STONE, la única variable que presentó asociación positiva con el tiempo entre la orden y la cirugía fue la presencia de hidronefrosis.

Conclusión La realización tardía de la ureterolitotomía láser puede tener un impacto en la morbilidad de los pacientes con patología litiásica, en especial en las complicaciones postoperatorias. Esto plantea una necesidad de priorización de los pacientes según su probabilidad de presentar complicaciones. received

October 12, 2016

accepted

March 13, 2017

published online

January 10, 2018
DOI https://doi.org/

10.1016/j.uroco.2017.03.007.

ISSN 0120-789X.

eISSN 2027-0119.
Copyright ( 2018 , Sociedad Colombiana License terms de Urología. Publicado por Thieme Revinter Publicações Ltda., Rio de Janeiro, Brazil. Todos los derechos reservados.

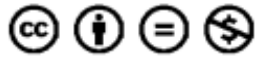




\begin{abstract}
Keywords

- laser lithotripsy

- flexible ureteroscopy

- urolithiasis

- complications

- risk factors

- risk assessment scale

Objectives The fibre-optics laser has become the surgical procedure of choice in most cases of urolithiasis over the years. However, there are few risk prediction scales described and in those that are published, they do not include the time delay in carrying out this procedure. The aim of this study is to present a description of the elements that influence the outcomes related to the complications of this surgery.

Materials and methods The study included 149 patients between 4 and 74 years who underwent flexible retrograde ureteroscopy with laser fragmentation technology between 2012-2015. The analysis was based on the outcomes related to the calculus features, anatomy, time between diagnosis and intervention, and surgery complications. The statistical analysis was performed using central tendency and measures of association.

Results A positive association was identified between preoperative, intraoperative, and in particular, in postoperative complications with the number of days between diagnosis and intervention, and with a positive trend that was related to the delay in the surgical intervention. As regards the STONE score, the only variable that had a positive association with the time between the request and performing the procedure, was the presence of hydronephrosis.

Conclusion The delay in the intervention time for this surgery in patients with urolithiasis could have an important impact on morbidity, especially for postoperative complications. These results suggest the need of prioritisation of patients, taking into account the prediction of complications.
\end{abstract}

\section{Introducción}

Uno de los mayores problemas que tienen los sistemas de salud en la actualidad es la dificultad en el acceso oportuno a tratamientos médicos y quirúrgicos. ${ }^{1}$ El acceso al manejo quirúrgico es completamente dependiente de los recursos dispuestos para ello y, a pesar de que se tiene poca información sobre cómo es el acceso, cuál es la calidad y que tan oportuno es en diferentes regiones del mundo, se sabe que el manejo tardío y poco oportuno de ciertas enfermedades insidiosas y prevalentes afectan negativamente a la calidad de vida de la población. Es el caso de la urolitiasis, lo que se evidencia en manejos quirúrgicos tardíos y su posterior impacto en morbimortalidad. ${ }^{2}$

En el caso específico de la litiasis urinaria se ha logrado un importante progreso en los últimos años, en especial con la innovación de la tecnología fibróptica láser. Desde la década de los 80 el desarrollo de técnicas quirúrgicas ha sido dirigida a la mínima invasión y a la solución de situaciones especiales en pacientes de alta comorbilidad, como la insuficiencia renal crónica y alteraciones anatómicas congénitas, entre otras. $^{3}$ Una de las situaciones más frecuentes para la utilización esta técnica es en pacientes con litiasis ubicadas en condiciones anatómicas desfavorables para otras técnicas y la falla con otros tratamientos quirúrgicos previos como la cirugía endoscópica convencional y litotripsia extracorpórea. La justificación para el desarrollo en la técnica se basa en menor tasa de lesiones intraoperatorias y menor necesidad de cirugías percutáneas y abiertas. ${ }^{4}$

Hay varios factores que han desencadenado un aumento de las listas de espera para la realización de esta cirugía, debido a que es un método poco invasivo y con bajas tasas de morbilidad asociadas, con acceso a lugares anatómicos en donde otras técnicas tienen dificultades y al aumento de las tasas de litiasis renal, no solo por el cambio en los patrones de alimentación y de calidad de vida, sino también por el mayor diagnóstico que ha sobrevenido gracias al avance de las técnicas radiológicas y diagnósticas dispuestas para ello. ${ }^{5}$

En el presente trabajo analizamos de manera retrospectiva el tiempo en días desde la orden de la cirugía hasta la realización de la misma en nuestra serie de ureterolitotomías endoscópicas (ULE), estudiando las tasas de complicación pre, intra y postoperatorias, y la necesidad de un segundo tiempo operatorio. Esto con el principal objetivo de hacer una aproximación sobre el efecto del sistema de salud en el pronóstico de estos pacientes, crear estrategias de predicción del riesgo quirúrgico, diseño de estrategias de priorización con objetivos en impacto de morbilidad, desenlaces desfavorables y costoeconómicos.

\section{Materiales y métodos}

Se diseñó un estudio descriptivo retrospectivo, en donde se incluyó a 149 pacientes con edades entre los 4 y los 74 años, que consultaron por patología urológica litiásica y fueron llevados a ULE entre los años 2012 y 2015, con los siguientes criterios de inclusión: paciente con indicación de realización de ULE con una intervención programada entre enero del 2012 y diciembre del 2015 que hayan sido intervenidos en el hospital Universitario San Ignacio, Clínica del Country o Clínica Colsubsidio. Los criterios de exclusión fueron: pacientes con litiasis de tipo coraliforme mayor 
a $20 \mathrm{~mm}$, con comorbilidades específicas como neoplasias, que se encontraran en tratamientos que indujeran inmunosupresión (radioterapia, quimioterapia o consumo crónico de corticoides) o malformaciones anatómicas del tracto genitourinario.

Previo al inicio de la recolección de datos mediante el método de revisión de historias clínicas, se realizó un formato de recolección las variables de interés a evaluar, dentro de las cuales se encontraban características sociodemográficas de los pacientes, como sexo y edad, características del cálculo como su tamaño, localización, lateralidad y densidad; factores quirúrgicos, como las complicaciones pre, intra y postoperatorias, el tiempo operatorio, la necesidad de intervenciones y derivaciones previas de la vía urinaria, la necesidad de colocación de catéter doble J posterior a la cirugía, la necesidad de reintervención y el tiempo en días desde la orden hasta la realización de la cirugía.

El proyecto fue aprobado por el comité de ética del Hospital Universitario San Ignacio; el formato fue sometido a una prueba piloto para evaluar su practicidad y estandarización con la posterior realización de correcciones adicionales. Se codificó la base de datos según posibles desenlaces y combinaciones de los mismos.

De acuerdo con la naturaleza de cada variable se hizo análisis de la población utilizando promedios y desviaciones estándar
(Z-score) para las variables continuas, para las variables nominales y ordinales descripción de frecuencias porcentajes y desviaciones estándar. Se evaluaron los datos por correlación en tablas dinámicas de Microsoft Excel $^{\circledR}$ (versión 15) y posteriormente se analizaron por medio de gráficas.

\section{Resultados}

Entre el período comprendido entre el año 2012 y 2015, se halló a 158 pacientes a los que se les aplicaron criterios de inclusión y de exclusión, en donde 149 pacientes cumplieron los criterios y fueron analizados.

\section{Características de la población}

La distribución de los casos con respecto a sexo, edad, necesidad de intervención previa, derivación previa, tamaño y topografía del cálculo, obstrucción previa, cantidad de cálculos, lateralidad y densidad del cálculo son descritos en la - Tabla 1.

La mayoría de los pacientes están en un rango de edad entre los 41-60 años (18,79\% de la población), sin predominancia significativa de algún sexo (47,6\% de sexo femenino y $52,4 \%$ de sexo masculino).

Tabla 1 Características de los pacientes

\begin{tabular}{|c|c|c|}
\hline Característica $(\mathrm{N}=149)$ & Sí valor, N. ${ }^{\circ}$ total, N. ${ }^{\circ}(\%)$ & No valor, N. ${ }^{\circ}$ total, N. ${ }^{\circ}(\%)$ \\
\hline Sexo & & $\mathrm{N}=149(100)$ \\
\hline Femenino & $78 / 149(47,65)$ & NA \\
\hline Masculino & $71 / 149(52,35)$ & NA \\
\hline $\begin{array}{l}\text { Edad promedio/distribución Promedio } \\
\text { (mín. 4/máx. 74) }\end{array}$ & 46,6 años $\pm 14,91$ & $N=149(100)$ \\
\hline $0-20$ & $4 / 149(2,68)$ & NA \\
\hline $21-40$ & $48 / 149(32,21)$ & NA \\
\hline $41-60$ & $72 / 149(18,79)$ & NA \\
\hline $61-80$ & $25 / 149(16,78)$ & NA \\
\hline Intervenciones previas & & $\mathrm{N}=149(100)$ \\
\hline ULE & $26 / 149(17,45)$ & NA \\
\hline Litotripsia extracorpórea & $20 / 149(13,4)$ & NA \\
\hline Nefrolitotomía percutánea & $17 / 149(11,4)$ & NA \\
\hline Cistolitotomía endoscópica & $3 / 149(2)$ & NA \\
\hline Litotripsia neumática & $1 / 149(0,67)$ & NA \\
\hline Ureterolitotomía abierta & $1 / 149(0,67)$ & $\mathrm{NA}$ \\
\hline Ninguna & NA & $81 / 149(54,36)$ \\
\hline Derivación previa & $46 / 149(30,87)$ & $103 / 149(69,12)$ \\
\hline Tamaño del cálculo (mm) & $\begin{array}{l}\text { Promedio }=13,72 \pm 5,9 \text { (mín. 5/máx. } \\
37 \text { ) }\end{array}$ & $N=149(100)$ \\
\hline$<5$ & $0 / 149(0,00)$ & NA \\
\hline $5-9$ & $37 / 149(24,8)$ & NA \\
\hline $10-20$ & $98 / 149(65,77)$ & NA \\
\hline$>20$ & $10 / 149(6,7)$ & NA \\
\hline Sin datos & $4 / 149(2,6)$ & NA \\
\hline
\end{tabular}


70 Factores pronósticos médicos y sociales asociados a urolitiasis Quiñones y col

Tabla 1 (Continued)

\begin{tabular}{|c|c|c|}
\hline Característica ( $\mathrm{N}=149$ ) & Sí valor, N. ${ }^{\circ}$ total, N. ${ }^{\circ}(\%)$ & No valor, N. ${ }^{\circ}$ total, N. ${ }^{\circ}(\%)$ \\
\hline Topografía del cálculo (localización) & & $\mathrm{N}=149(100)$ \\
\hline Polo superior & $7 / 149(4,6)$ & NA \\
\hline Polo medio & $10 / 149(6,7)$ & NA \\
\hline Polo inferior & $15 / 149(10)$ & NA \\
\hline Pelvis renal & $10 / 149(6,7)$ & NA \\
\hline Unión pieloureteral & $7 / 149(4,6)$ & NA \\
\hline Uréter proximal & $87 / 149(58,3)$ & NA \\
\hline Uréter medio & $4 / 149(2,6)$ & NA \\
\hline Uréter distal & $6 / 149(4)$ & NA \\
\hline Coraliforme & $3 / 149(2)$ & NA \\
\hline Obstrucción (hidronefrosis) & $38 / 149(25,5)$ & $111 / 149(74,5)$ \\
\hline $\begin{array}{l}\text { Cantidad de cálculos (único/múltiples). } \\
\text { Múltiple (sin datos 46) }\end{array}$ & $\begin{array}{l}N=103(100) \\
15 / 103(14,6)\end{array}$ & $\begin{array}{l}\text { (Únicos) } \\
88(85,4)\end{array}$ \\
\hline Densidad de los cálculos (UH) & Promedio $=1.007,5 \pm 376$ & $\mathrm{~N}=55(100)$ \\
\hline$<750$ & $13 / 65(20)$ & NA \\
\hline $750-1.000$ & $20 / 65(30,76)$ & NA \\
\hline$>1.000$ & $32 / 65(49,23)$ & NA \\
\hline Sin datos (84) & $84 / 149(63,63)$ & NA \\
\hline Complicaciones preoperatorias & $56 / 149(37,58)$ & $\mathrm{N}=149(100)$ \\
\hline Hidronefrosis & $38 / 149(25,5)$ & NA \\
\hline $\begin{array}{l}\text { Infección de vías urinarias (IVU, } \\
\text { pielonefritis y sepsis) }\end{array}$ & $11 / 149(7,38)$ & NA \\
\hline Lesión renal aguda & $3 / 149(2,01)$ & NA \\
\hline Catéter JJ calcificado & $3 / 149(2,01)$ & NA \\
\hline $\begin{array}{l}\text { Otras (Estrecheces, malformaciones } \\
\text { anatómicas) }\end{array}$ & $1 / 149(0,67)$ & NA \\
\hline Ninguna & NA & $93 / 149(62,4)$ \\
\hline Complicaciones intraoperatorias & $12 / 149(8,05)$ & $N=149(100)$ \\
\hline Esfacelación ureteral & $6 / 149(4,02)$ & NA \\
\hline Perforación de la vía urinaria & $3 / 149(2,01)$ & NA \\
\hline $\begin{array}{l}\text { Sangrado complicado (pendiente } \\
\text { revisar historia) }\end{array}$ & $2 / 149(1,34)$ & NA \\
\hline Imposibilidad de abordaje & $1 / 149(0,67)$ & NA \\
\hline Ninguna & NA & $137 / 149(91,94)$ \\
\hline Complicaciones postoperatorias & $16 / 149(10,73)$ & $\mathrm{N}=149(100)$ \\
\hline Fiebre sin foco transitoria & $4 / 149(2,68)$ & NA \\
\hline Infección de vías urinarias y Sepsis & $10 / 149(6,7)$ & NA \\
\hline $\begin{array}{l}\text { Pielonefritis + hematoma perirrenal } \\
\text { sobreinfectado }\end{array}$ & $1 / 149(0,67)$ & NA \\
\hline Hidronefrosis & $1 / 149(0,67)$ & NA \\
\hline Ninguna & NA & $133 / 149(89,26)$ \\
\hline Tiempo orden-procedimiento (días) & $\begin{array}{l}\text { Promedio }=65,89 \pm 44,32 \text { (mín. 0/ } \\
\text { máx. 285) }\end{array}$ & $N=132(100)$ \\
\hline 0.50 & $55 / 132(41,66)$ & NA \\
\hline 1-30 días & $21 / 132(15,9)$ & NA \\
\hline
\end{tabular}


Tabla 1 (Continued)

\begin{tabular}{|c|c|c|}
\hline Característica $(\mathrm{N}=149)$ & Sí valor, $\mathrm{N}^{\circ} /$ total, $^{\mathrm{N}} .^{\circ}(\%)$ & No valor, N..$^{\circ}$ total, N. ${ }^{\circ}(\%)$ \\
\hline 31-50 días & $34 / 132(25,75)$ & NA \\
\hline $51-100$ & $57 / 132(43,18 \%)$ & NA \\
\hline $101-150$ & $13 / 132(9,84)$ & NA \\
\hline $151-200$ & $4 / 132(3,03)$ & NA \\
\hline$>200$ & $3 / 132(2,27)$ & NA \\
\hline \multicolumn{3}{|l|}{ Sin datos $17 / 149(11,4)$} \\
\hline Catéter IJ posprocedimiento & $32 / 149(21,47)$ & $117 / 149(78,52)$ \\
\hline Catéter JJ retirado en seguimiento & $103 / 117(88)$ & $14 / 117(11,9)$ \\
\hline $\begin{array}{l}\text { Cálculo residual en radiografía de control } \\
\text { (N/datos faltantes } 21(14 \%)\end{array}$ & $49 / 128(38,28)$ & $\begin{array}{l}\mathrm{N}=128(100) \\
79 / 128(61,71)\end{array}$ \\
\hline Necesidad de un segundo tiempo & $16 / 149(10,73)$ & $133 / 149(89,26)$ \\
\hline
\end{tabular}

Los casos de promedios se presentan como media \pm desviación estándar.

En negrita, se muestran, de arriba abajo, el rango de edad en el que se encuentran la mayoría de los pacientes; la localización en donde se encontraban la mayoría de los cálculos que fueron llevados a ULE, y la principal complicación de los pacientes sometidos a ULE en este estudio.

La mayoría de los pacientes no requirieron una derivación previa de la vía urinaria (69,1\%), mientras que el 30,8\% sí la requirieron. Con relación a las características del cálculo, se incluyeron variables relacionadas con la escala STONE, ${ }^{6}$ como el tamaño del cálculo, que en la mayoría de los pacientes estuvo entre $10-20 \mathrm{~mm}$ (65.77\%); la localización más frecuente del cálculo fue en el uréter proximal (58,3\%), seguido de la localización en polo inferior (10\%). Las otras localizaciones tuvieron una distribución similar.

\section{Desenlaces quirúrgicos y características administrativas}

Con relación a las complicaciones preoperatorias, la mayoría de estas fueron hidronefrosis (25,5\%) y en segundo lugar infección de vías urinarias (7,38\%). Las complicaciones intraoperatorias se presentaron en un 8,05\% de los pacientes, dentro de los cuales la mayoría presentó una esfacelación ureteral (4,02\%) y las complicaciones postoperatorias se presentaron en un 10,73\% de los pacientes, en los que la principal complicación fue la infección y sepsis de vías urinarias.

A la mayoría de los pacientes intervenidos se les derivó la vía urinaria con un catéter doble J (78,52\%), de los cuales solo fueron retirados durante el seguimiento al $88 \%$ de los pacientes. Las radiografías de control demostraron cálculos residuales en el $38,28 \%$ de los casos. El segundo tiempo quirúrgico, de los cuales solo fueron tenidos en cuenta en las intervenciones ipsolaterales, fue necesario en el 10,73\% de los pacientes.

Se correlacionaron las variables de complicaciones quirúrgicas pre, intra y postoperatorias con el tiempo ordenprocedimiento y se encontró una asociación positiva ascendente en todas las anteriores. Con las complicaciones preoperatorias se evidencia una relación positiva ( - Fig. 1), de la misma forma en las complicaciones intraoperatorias ( - Fig. 2) y finalmente en las postoperatorias, con una tendencia porcentual ascendente fuertemente positiva entre más tardíamente se realiza la cirugía, con una pendiente de 0,93 (-Fig. 3).

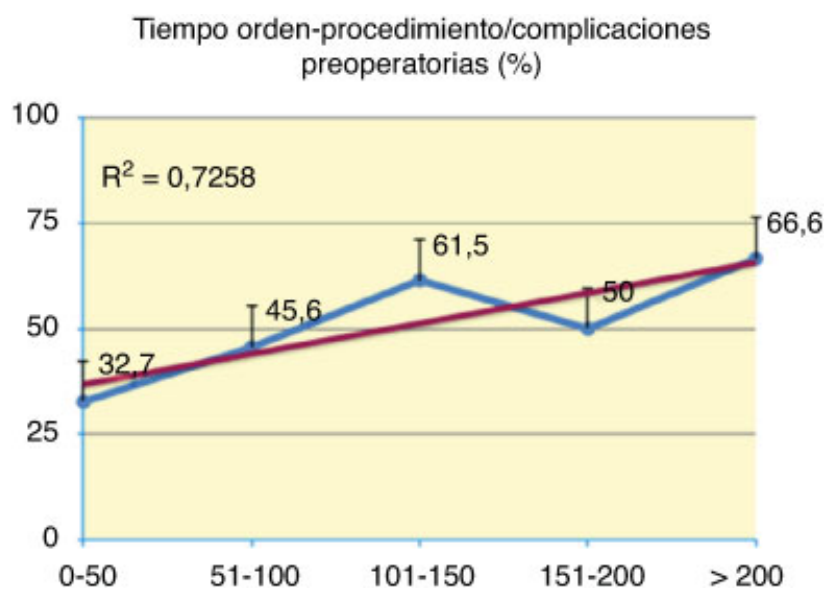

Fig. 1 Relación entre el tiempo en días desde la emisión de la orden quirúrgica hasta la realización de la ureterolitotomía láser y el porcentaje de las complicaciones preoperatorias.

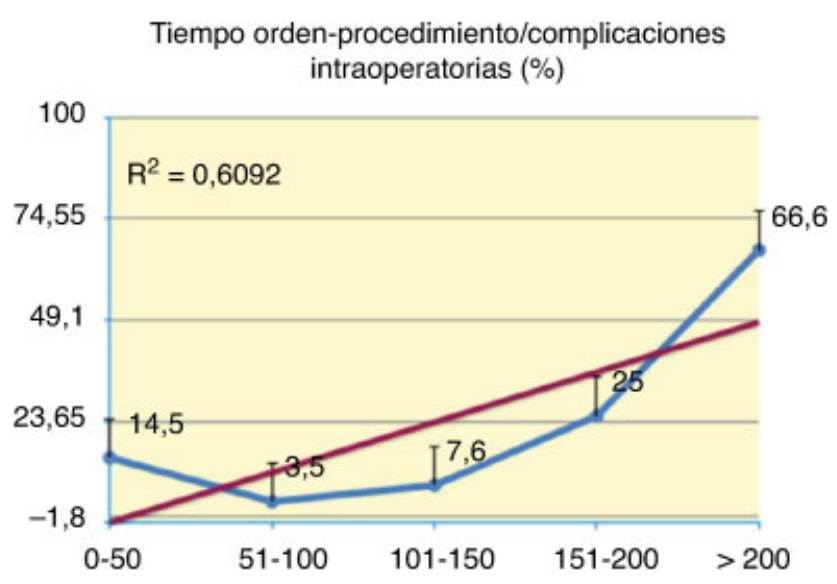

Fig. 2 Relación entre el tiempo en días desde la emisión de la orden quirúrgica hasta la realización de la ureterolitotomía láser y el porcentaje de las complicaciones intraoperatorias. 


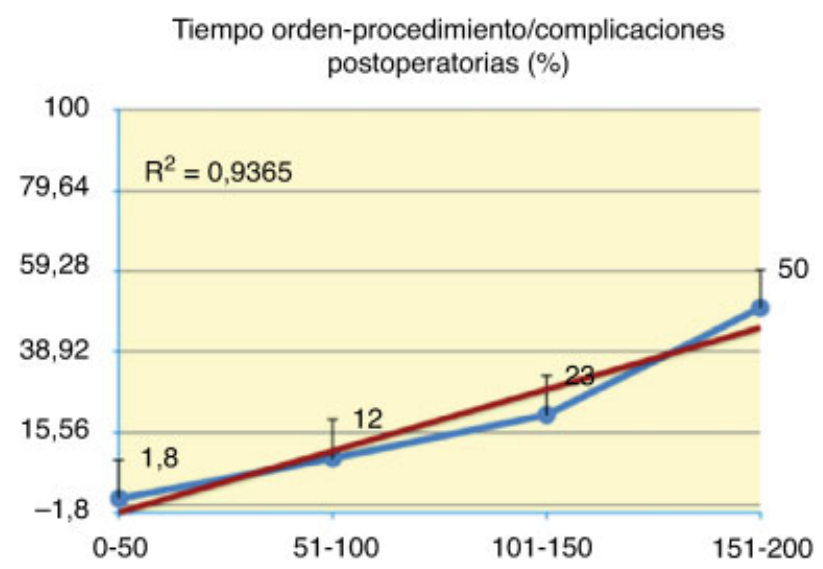

Fig. 3 Relación entre el tiempo en días desde la emisión de la orden quirúrgica hasta la realización de la ureterolitotomía láser y el porcentaje de las complicaciones postoperatorias.

Adicionalmente, se encontró una pendiente ascendente al asociar el tiempo orden-procedimiento con la necesidad de un segundo tiempo operatorio, mostrando una correlación entre el tiempo en que se posterga la cirugía con un aumento en la necesidad del segundo tiempo de manera proporcional (-Fig. 4).

Al asociar las variables del índice STONE con el tiempo orden-procedimiento se evidencia que para el diámetro del cálculo no encontró asociación entre este y el tiempo entre la orden y el procedimiento; en la densidad del cálculo tampoco se evidenció una relación ascendente. En el caso de la presencia de hidronefrosis, se evidencia un aumento significativo de los casos con relación a un tiempo ordenprocedimiento más prolongado.

Con respecto a la relación entre el tiempo operatorio y el tiempo orden-cirugía, se halló una correlación positiva en donde a medida que se intervenía el paciente más tardíamente, se presentaban tiempos operatorios más prolongados, como se observa en la - Figura 5, con un tiempo promedio de cirugía de $96,8 \mathrm{~min}$.

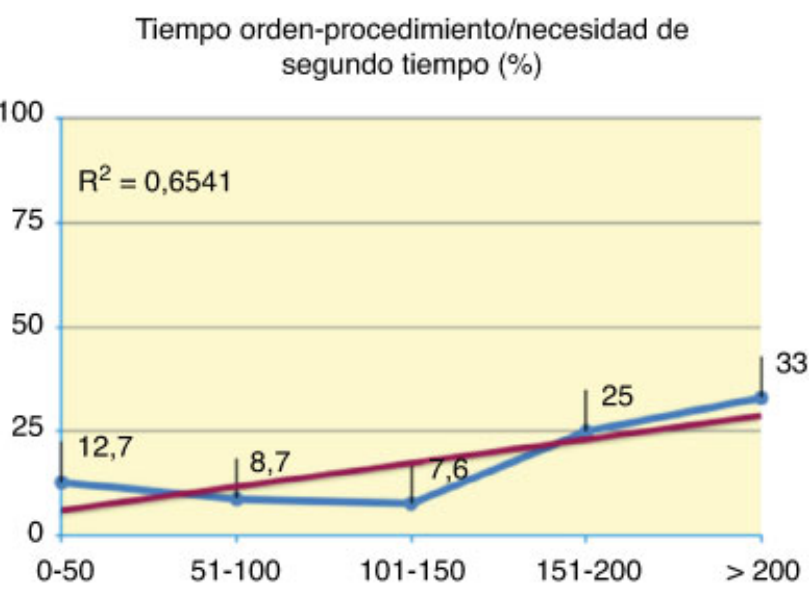

Fig. 4 Relación entre el tiempo en días desde la emisión de la orden quirúrgica hasta la realización de la ureterolitotomía láser y la necesidad de un segundo tiempo operatorio.
Tiempo orden-procedimiento/promedio tiempo operatorio

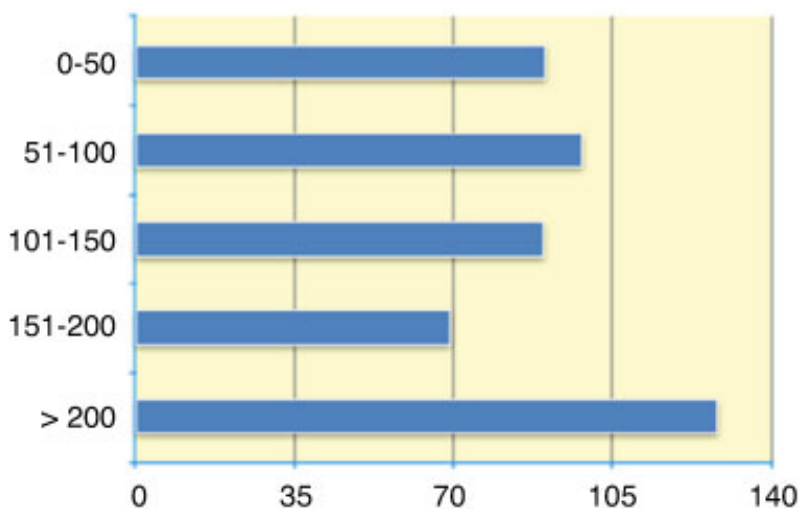

Fig. 5 Relación entre el tiempo en días desde la emisión de la orden quirúrgica hasta la realización de la ureterolitotomía láser y el tiempo operatorio (en minutos).

\section{Discusión}

Se han descrito en la literatura escalas e índices que proponen factores predictores de complicaciones en la población sometida a cirugía, como la escala de Clavien, ${ }^{7}$ y algunas específicas para urolitiasis, como el índice STONE, ${ }^{6}$ que predice este riesgo mediante las características intrínsecas del cálculo. Sin embargo, en ningún caso se han tenido en cuenta factores como la intervención temprana vs. la intervención tardía de estos pacientes y su relación con las complicaciones pre, intra y postoperatorias, con la necesidad de reintervención y con la duración del tiempo operatorio.

En la presente investigación hallamos que existe una distribución demográfica y de características de los pacientes similar a la de otras poblaciones, ${ }^{8}$ sin una predominancia de sexo y con una distribución relativamente igualitaria con respecto a la edad. Aproximadamente, la mitad de los pacientes habían sido sometidos a una intervención previa (45\%), de las cuales la principal intervención previa fue otra ULE (17,5\%), lo cual nos muestra que es una de las intervenciones con más recurrencias, aunque hay que tener en cuenta que este porcentaje también se ve influenciado porque es una de las cirugías más realizadas.

Aunque en la mayoría de los pacientes no había derivado la vía urinaria previamente, existe un porcentaje importante en los que sí (30\%), similar al de los casos de los pacientes que desarrollaron hidronefrosis previamente a la ULE (25,5\%). Con respecto a las características del cálculo, la mitad de estos tenían más de 1.000 unidades Hounsfield, lo que adicionalmente da una dificultad mayor para su fragmentación.

Por los hallazgos descritos, en el apartado de resultados se sugiere que puede existir una relación entre la intervención tardía de los pacientes que son llevados a ULE con la incidencia de las complicaciones pre, intra y postoperatorias, lo que hace que el tiempo de realización de la cirugía sea un potencial predictor de peor pronóstico para estos pacientes. Existen variables que se correlacionan con un mayor tiempo de latencia entre la orden y el procedimiento, entre estas el tiempo operatorio y la necesidad del segundo tiempo, lo 
cual determina que, a pesar de la experticia del equipo de cirujanos, pueden ser factores predictores de complicación.

Es importante resaltar que pueden existir otras asociaciones adicionales con una mayor tasa de complicaciones a las previamente mencionadas, como, por ejemplo, el número de intervenciones previas a la ULE, las características conformacionales del cálculo y un tiempo prolongado en el retiro del catéter doble J, entre otras. Adicionalmente, en este estudio se encontró que existe una pérdida de seguimiento de algunos pacientes a los que se les inserta un catéter de derivación doble J, lo cual es preocupante debido a que se ha demostrado en estudios previos que la calcificación ocurre luego de que este permanezca en la vía urinaria durante períodos prolongados, generalmente 12 semanas o más. ${ }^{9}$

Con lo hallado anteriormente, consideramos que es importante tener un método de priorización para los pacientes y no simplemente programar las cirugías por "orden de llegada" en el momento de realizar el diagnóstico de urolitiasis. Esto debido a que en los métodos de priorización se garantiza la clasificación justa y basada en la clínica, así como la disminución del tiempo de espera para la realización de la misma. ${ }^{10}$ Los desenlaces son mejores resultados quirúrgicos, mayor optimización de la actividad y disponibilidad el personal especializado, efectividad del tratamiento, así como la reducción de costos en hospitalizaciones por complicaciones y costos por reprogramación, además de una mejoría en las tasas de correlación entre la oferta y demanda de las instituciones. ${ }^{11,12}$

\section{Perspectivas}

Se considera que se deberían realizar más publicaciones para poder demostrar las hipótesis mencionadas en el presente estudio, en especial la relación causal entre una intervención quirúrgica tardía -en el caso de la ULE-, con una mayor tasa de complicaciones pre, intra y postoperatorias, con el fin de desarrollo de escalas de riesgo para poder tener una priorización de los pacientes con mayor probabilidad de tener complicaciones, y evitar tiempos de espera para el procedimiento prolongados, y consecuentemente evitar complicaciones.

Se resalta que los especialistas son los encargados de referir a los pacientes a listas de espera, que hay pocos criterios y acuerdo para los médicos especialistas tomar decisiones, existen diferencias entre las instituciones. Se considera las estrategias de priorización como forma de hacer la cirugía más igualitaria. El sistema de priorización se debe basar en aspectos claves, igualdad y transparencia, certeza y sobre todo herramientas validadas científicamente para priorización, las cuales deben ser simples de usar, tener en cuenta todos los criterios importantes que puedan influir en la prioridad de la cirugía (factores predictores de complicación), influir en decisiones críticas, dar un puntaje cuantitativo que den un punto de acuerdo en toma de decisiones y ser reproducibles.

\section{Responsabilidades éticas}

\section{Protección de personas y animales}

Los autores declaran que para esta investigación no se han realizado experimentos en seres humanos ni en animales.

\section{Confidencialidad de los datos}

Los autores declaran que han seguido los protocolos de su centro de trabajo sobre la publicación de datos de pacientes.

\section{Derecho a la privacidad y consentimiento informado} Los autores declaran que en este artículo no aparecen datos de pacientes.

Conflicto de intereses

Los autores declararon no tener conflictos de interés.

\section{Bibliografía}

1 Derose KP, Gresenz CR, Ringel JS. Understanding disparities in health care access and reducing them through a focus on public health. Health Aff 2011;30:1844-1851

2 Rogers SO. Disparities in surgery: Access to outcomes. World J Surg 2008;32:505-508

3 Alcaide JR.C, Togores LH, Castillo PM.C, Maestro MA, Aguilera A, Codes FR.D. Tratamiento de las litiasis con láser en condiciones especiales. Arch Esp Urol 2008;9:111-114

4 Redondo C, Ramón de Fata F, Gimbernat H, Meilán E, Andrés G, Angulo JC. Retrograde intrarenal surgery with holmium-YAG laser lithotripsy in the primary treatment of renal lithiasis. Actas Urológicas Españolas (English Ed [Internet] 2015;39:320-326 [consultado 16 Feb 2016]. Disponible en: http://linkinghub. elsevier.com/retrieve/pii/S2173578615000669

5 Richman K, O'Bell JG.P. The growing prevalence of kidney stones and opportunities for prevention. R I Med J 2013;97:31-34

6 Molina WR, Kim FJ, Spendlove J, Pompeo AS, Sillau S, Sehrt DE. The S.T.O.N.E. Score: A new assessment tool to predict stone free rates in ureteroscopy from pre-operative radiological features. Int Braz J Urol [Internet] 2014;40:23-29 [consultado 16 Feb 2016]. Disponible en: http://www.ncbi.nlm.nih.gov/pubmed/24642147

7 Dindo D, Demartines N, Clavien P-A. Classification of surgical complications. Ann Surg [Internet] 2004;240:205-213 [consultado 24 Feb 2016]. Disponible en: http://content.wkhealth.com/linkback/ openurl?sid=WKPTLP:landingpage $\& a n=00000658-200408000-$ 00003

8 De la Rosette J, Denstedt J, Geavlete P, Keeley F, Matsuda T, Pearle $\mathrm{M}$, et al. The clinical research office of the endourological society ureteroscopy global study: Indications, complications, and outcomes in 11,885 patients. J Endourol [Internet] 2014; 28:131-139 [consultado 3 Mar 2016]. Disponible en: http:// www.ncbi.nlm.nih.gov/pubmed/24147820

9 Zisman A, Siegel YI, Siegmann A, Lindner A. Spontaneous ureteral stent fragmentation. J Urol 1995;153:718-721

10 Abásolo I, Barber P, González López-Valcárcel B, Jiménez O. Real waiting times for surgery. Proposal for an improved system for their management. Gac Sanit [Internet] 2014;28:215-221 [consultado 3 Mar 2016]. Disponible en: http://www.sciencedirect.com/science/ article/pii/S0213911113002264

11 Curtis AJ, Russell CO.H, Stoelwinder JU, McNeil JJ. Waiting lists and elective surgery: Ordering the queue. Med J Aust 2010;192:217-220

12 Dimakou S, Dimakou O, Basso HS. Waiting time distribution in public health care: empirics and theory. Health Econ Rev [Internet] 5;2015:10-14 [consultado 3 Mar 2016]. Disponible en: http://dx.doi.org/10.1186/s13561-015-0061-7 